\title{
Fractional Stochastic Individuals
}

\author{
M. Turalska ${ }^{1}$ and B. J. West ${ }^{1,3}$ \\ 1) Physics Department, Duke University, Durham, NC \\ 2) Information Sciences Directorate, Army Research Office, \\ Research Triangle Park, NC 27709
}

May 16, 2013

\begin{abstract}
The decision making model (DMM) [1, 2] has been shown to generate phase transitions, to be topologically complex and to manifest temporal complexity through size-dependent random fluctuations in the switching times between the two critical states of consensus. These properties are entailed by the fundamental assumption that the network elements in the DMM imperfectly imitate one another. The process of subordination establishes that a single network element can be described by a fractional master equation whose analytic solution yields the observed autocorrelation function obtained by numerical integration of the DMM to a high degree of accuracy.
\end{abstract}

\section{Introduction}

Fractional differential equations have been found to be a convenient way to describe the dynamics of complex phenomena that are characterized by multiple scales, that is, to describe the time evolution of fractal processes [3] with no single scale being dominant. In spite of the success of the mathematical descriptions of such processes there has been a lack of identification and interpretation of mechanisms that entail fractional dynamic equations in a social context. Herein we provide an explanation of one source of a fractional differential equation that describes the dynamics of a complex network using a fractional master equation.

West and Turalska [4] adopted imitation as a basic social/psychological mechanism that is part of what it means to be human. Imitation as a mechanism for explaining social behavior dates back to the turn of the twentieth century $[5,6,7]$ and is used [4] to construct a complex dynamic social network. In this social network the hypothesis that imperfect imitation (termed an echo response) is a fundamental mode of human behavior is made and implemented through the choice of interaction coefficients in the mathematics of the decision making model (DMM) $[1,2,8]$. The resulting dynamics were then used to determine what properties emerged as a consequence of the echo response hypothesis. 
It was shown that the hypothesis entails behavior, such as the phenomenon of consensus, that is not implicit in the initial statement of the model. In other words the hypothesis was implemented through the choice of the coupling coefficients in the DMM and the resulting critical dynamics was used to explain such collective effects as herding, flocking and schooling as well as manifesting stochastic behavior. The DMM is sketched out in Section 2.

Stochastic differential equations have historically been derived in physical systems using Hamiltonian-based arguments in which the system of interest is coupled to an infinitely large environment. These derivations produce Langevin equations, which involve coarse graining the dynamic description so that the influence of the environment appears in the equation of motion as a linear dissipation and random fluctuations that are interdependent through a fluctuationdissipation relation [9]. This technique basically decomposes the system into macroscopic and microscopic time scales with the later providing the random fluctuations associated with thermal effects. More recently it was shown that when the microscopic dynamics are given by a non-integrable Hamiltonian the separation of time scales no longer occurs because the microscopic time scales diverge to macroscopic size, resulting in a fractional Langevin equation for the system dynamics [10]. Herein we find a similar result, in the sense that the dynamics of the smallest component of the complex network, the individual, can be described by a fractional Langevin equation as shown in Section 3. However in the present approach no coarse graining is envoked.

In the DMM neither the single individual nor the dynamics of the social network are specified by Hamiltonians. The predicted behavior of the single element dynamics is compared with the numerical results from the DMM implemented on a two-dimensional lattice in Section 4. The influence of the network on the dynamics on the individual is measured by the individual autocorrelation function. The autocorrelation function is shown to be a stretched exponential in the subcritical region, to be a modulated stretched exponential in the supercritical domain and a modulated Mittag-Leffler function in the critical region. In all three regions the analytic solutions to fractional Langevin equations provide excellent fits to the numerical calculations of the complex network.

In Section 5 some conclusions are drawn.

\section{Decision Making Model (DMM)}

The DMM of a complex social network represents the dynamics of the probability for an individual to be in either of two states, yes or no, up or down, on or off, by a set of coupled two-state master equations. Turalska et al. $[2,8]$ showed that this set of equations for a complex network has the same structural form as that of the individual elements of the network:

$$
\begin{aligned}
& \frac{d}{d t} p_{1}=-g_{12} p_{1}+g_{21} p_{2}, \\
& \frac{d}{d t} p_{2}=-g_{21} p_{2}+g_{12} p_{1} .
\end{aligned}
$$


The quantity $p_{j}(t)$ is the probability of the network being in the state $j=1,2$ at time $t$ and the probability is normalized at all times such that

$$
p_{1}(t)+p_{2}(t)=1 .
$$

The dynamics are determined by the choice of the functional form of the transition rates in Eqs.(1) and (2). In this first case each probability is influenced by the states occupied by all the elements of the network as determined by the transition rates

$$
g_{i j}(t)=g_{0} \exp \left[K\left\{\Sigma_{j}(t)-\Sigma_{i}(t)\right\}\right] ; i \neq j=1,2
$$

where

$$
\Sigma_{s}(t)=\frac{N_{s}(t)}{N} .
$$

$N$ denotes the total number of elements in the network and $N_{s}(t)$ is the number of elements in the state $s=1,2$ at time $t$. The parameter $K$ is the control parameter that determines the strength of the interactions between elements of the network. It is evident that the quantity $\Sigma_{s}$ is an erratic function of time, and is a global property, obtained from an observation of the entire network. We define the stochastic global variable

$$
\xi(t) \equiv \Sigma_{1}(t)-\Sigma_{2}(t)=\frac{N_{1}(t)-N_{2}(t)}{N},
$$

whose variability is characteristic of the entire network of echoes, that is, the echoing response of the network to the echoed opinions of its coupled constituents.

In the situation where the number of nearest neighbors coupled to the element of interest consists of all the other individuals in the network we have all-to-all (ATA) coupling. Consider the ATA coupling case and assume that the total number of elements within the network $N$ becomes infinite. In the $N \rightarrow \infty$ case the fluctuation frequencies collapse into probabilities according to the law of large numbers $\Sigma_{s}=p_{s}$. In physics this replacement goes by the name of the mean field approximation in which case the transition rates in the $\mathrm{ME}$ are written

$$
g_{i j}=g_{0} \exp \left[-K\left(p_{i}-p_{j}\right)\right] ; i \neq j=1,2 .
$$

The formal manipulation of the master equation even in this simplified case is made a little simpler if we introduce the difference in the probabilities

$$
\Pi \equiv p_{1}-p_{2} .
$$

Subtracting Eq. (2) from Eq. (1) after some algebra yields the highly nonlinear rate equation for the difference variable

$$
\frac{d}{d t} \Pi=-\left(g_{12}+g_{21}\right) \Pi+\left(g_{21}-g_{12}\right)
$$


where the nonlinearity enters through the transition rate dependence on the difference variable

$$
g_{12}=g_{0} \exp [-K \Pi] ; g_{21}=g_{0} \exp [K \Pi]
$$

in the mean field approximation. By inserting Eq. (10) into Eq. (9) we obtain

$$
\frac{d}{d t} \Pi=-\frac{\partial V}{\partial \Pi}
$$

and the network dynamics are determined by the potential function $V(\Pi)$, which is a symmetric double well potential with the explicit form

$$
V(\Pi)=\frac{2 g_{0}}{K}\left[\Pi \sinh K \Pi-\frac{K+1}{K} \cosh K \Pi\right] .
$$

Note that the social network is not described by a Hamiltonian and yet the global dynamics is indeed described by an effective Hamiltonian, that being the double well potential.

The cooperative behavior of the infinitely large ATA coupled network described by Eq.(11) is that of an overdamped particle hopping from one potential minimum to the other, whose position is $\Pi$ within the potential Eq.(12). For $K<1$, half of the nodes are in the state 1 and half are in the state 2 because there is only a single broad minimum in the potential. At the critical value of the control parameter $K=K_{c}=1$ a bifurcation occurs and the potential develops two wells separated by a barrier as discussed by Turalska et al. [2]. The height of the barrier increases with the value of the control parameter.

It is interesting that at the critical value of the control parameter the ATA version of the DMM undergoes a phase transition. Note that the amplitude of $\xi(t)$ depends on the value of the control parameter $K$. When $K=0$, all elements in the network are independent Poisson processes; thereby an average taken at any moment of time over all of them yields zero. Once the value of the coupling becomes nonzero, $K>0$, single elements are less and less independent, resulting in nonzero averages. The quantity $K_{c}$ is the critical value of the control parameter $K$, at which point a phase transition to a global majority state occurs. In numerical calculations we use the time average $\xi_{e q}=\langle|\xi(t)|\rangle$ as a measure of this global majority. More precisely, after an initial $10^{6}$ time steps, the average is taken over the same number of the consecutive time steps of the model. In Figure 1 the thin line indicates the ATA phase transition as measured by $\xi_{e q}$. The other phase transitions indicated are for the Ising model (dashed line) and the DMM model on a two-dimensional lattice as discussed elsewhere [8].

Real-world networks are not ATA coupled since interactions typically have finite range and elements are spatially separated. Moreover, real-world networks have finite numbers of elements. It is therefore useful to examine how strongly the mean field solutions are violated when we relax these constraints. The stability condition can be violated in at least two different ways. The first way is by reducing the number of elements $N$ to a finite value. The second way is by restricting the number of links so the network no longer has ATA coupling. 


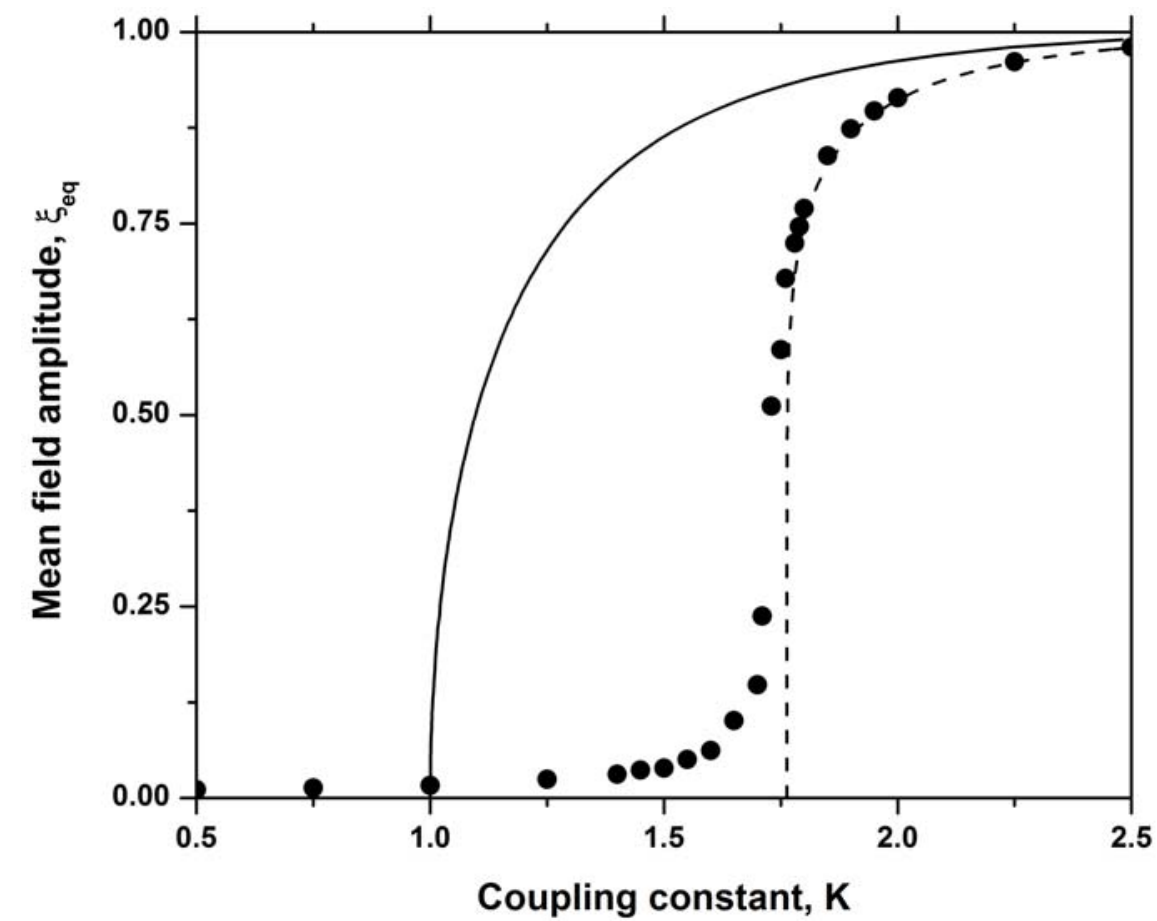

Figure 1: The phase diagram for the global variable $\xi_{\text {eq }}$. The thin solid line and the dashed line are the theoretical predictions for the fully connected and the two-dimensional regular network, respectively. In both cases $N=\infty$ and the latter case is the Onsager theoretical prediction [?] for a two-dimensional regular lattice. The dots corresponds to the global states observed for the DMM on a two-dimensional regular lattice $(N=100 \times 100$ nodes $)$ and $g_{0}=0.01$. Periodic boundary conditions were applied in the DMM calculations. (From [2] with permission.)

In real-world networks both sources of equilibrium disruption are expected to occur. For the time being we retain the ATA coupling within the network and consider the number of elements $N$ to be finite. In this latter case we can no longer make the mean field approximation and the dynamic picture stemming from the above master equation is significantly changed.

If the number of elements is still very large, but finite, we consider the mean-field approximation to be nearly valid and replace the deterministic equation Eq.(11) with the variable $\Pi$ replaced by the global variable $\xi$ to obtain a stochastic differential equation $[1,2]$ :

$$
\frac{d \xi(t)}{d t}=-\frac{\partial V(\xi)}{\partial \xi}+\varepsilon(t)
$$


and the additive fluctuations $\varepsilon(t)$ have amplitudes that are computationally determined to be on the order of $1 / N$. Here again we see the influence of the imperfect imitations that echo between individuals in the network.

Note that the double-well potential in the mean field approximation persists in the present description. The random fluctuations induce transitions between the two states of the potential well. Consequently, for a network with a finite but large number of elements the phase synchronization of Eq.(11) is not stable and the stochastic differential equation represents the dynamics of the network that must be solved. Furthermore the fluctuations can drive the particle from one well of the potential to the other when its amplitude is sufficient to traverse the barrier separating the wells. However, here the fluctuations arise from the finite number of elements in the network rather than from non-existent thermal excitations and are consequently suppressed as the network size increases.
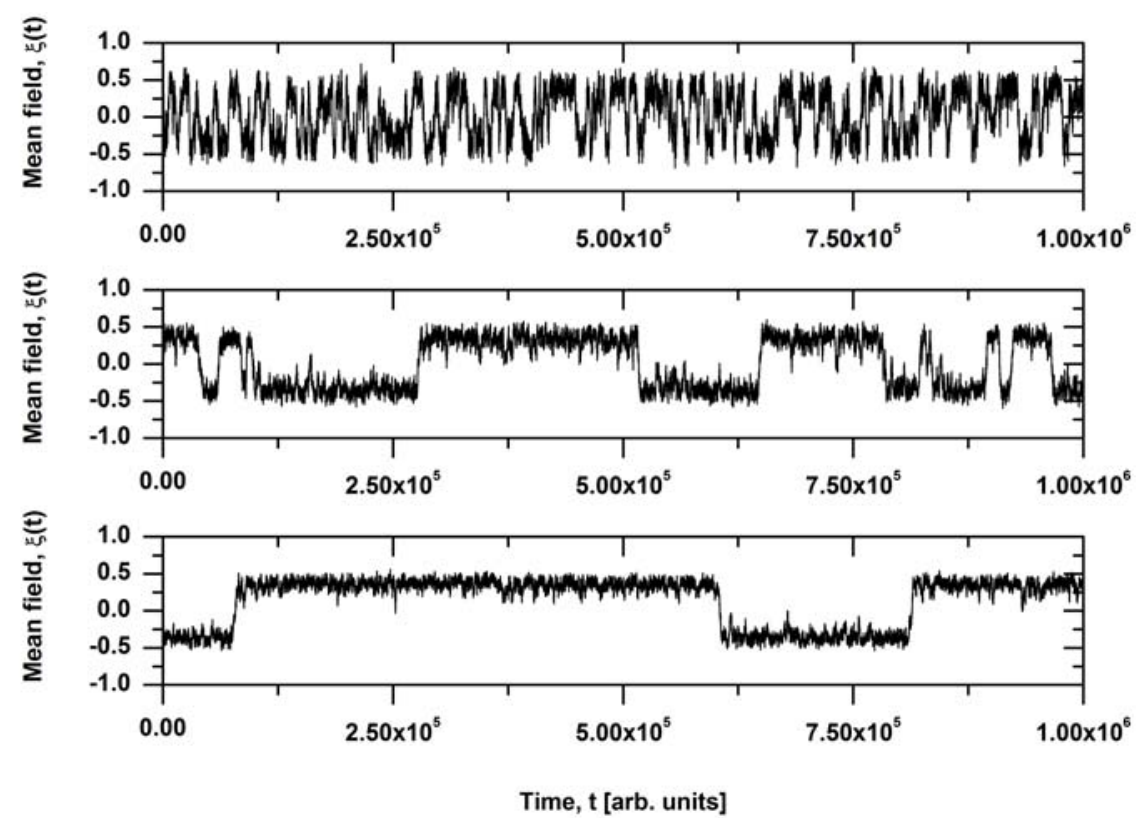

Figure 2: The fluctuation of the mean field-average phase as a function of time. For a system of $N=500$ elements (top), $N=1500$ elements (middle), and $N=2500$ elements (bottom). The coupling constant is $K=1.05$ and $g=0.01$ in all three cases. (From [2] with permission.)

Although Eq. (13) is written in the continuous time representation, in practice the numerical calculations of DMM correspond to the adoption of a finite integration time step $\Delta t=1$. Note that the stochastic rate equation Eq.(13) replaces Eq. (11) in the case of a finite $N$, and that Eq. (11) is recovered in the ideal case $N=\infty$. Consider the ATA coupling condition with a finite number 
of elements by numerically integrating the master equation for each element in the network and then calculating the number of elements in each of the two states. In Figure 2 the fluctuating global variable $\xi(t)$ is depicted as a function of time, under differing conditions. Notice that with increasing $N$ the fluctuation $\xi(t)$ become more distinctly dichotomous-like, with an increasingly sharp transition from the 'up' to the 'down' state. This pattern corresponds to the entire network keeping a decision for a longer and longer time as the size of the network increases. The condition of a decision lasting forever is reached in the ideal case $N=\infty$. The global variable fluctuates between the two minima of the double-well potential as described by Eq.(13) for $K=1.05>K_{c}$ and three values of the size of the network corresponding to an ever increasing influence of the echo network. The single element follows the fluctuations of the global variable, switching back and forth from the condition where the +1 state is preferred statistically to that where the -1 sate is preferred statistically. The complete properties of the ATA DMM are explored by Turaska et al. $[2,8]$.

\section{Subordination and Fractional Dynamics}

Note that if attention is concentrated on a single network element when the network is in a consensus state that individual would still appear to make transitions according to an exponential distribution as suggested by Figure 3. That is the situation on a large scale view of the calculations on a two-dimensional lattice with nearest neighbor interactions and a critical control parameter value of $K_{c}=1.7$. The strict exponential is indicated by the black dotted curve. The single particle survival probabilities do not look too much different, the light gray dashed curve with the subcritical value $K=1.5<K_{c}$ is very close to the exponential. The remaining single particle curves, whether critical $K=K_{c}=1.7$ or supercritical $K>K_{c}$ appear to be exponential on this graph. The difference in the behavior of the individual from that in the non-interacting state would be that she tends to be more reluctant to change her mind.

The deviation of the individual survival probability from the exponential form appears to be modest when compared with the dramatically greater deviation of the survival probability of the global variable from the exponential. The average network behavior differs markedly as the control parameter is increased from the subcritical through the supercritical regions. However the influence of the global variable on the behavior of the individual does not appear to induce a significant change. For the individual the change is a subtle yet profound difference and is a direct result of the hypothesized imitation mechanism. So if the individual survival probability is not exponential, what is it? To answer

this question we turn our attention to describing an alternate construction of the individual element dynamics.

The master equation for a single isolated element is according to the numerical simulation given by

$$
\phi(n \Delta \tau)-\phi([n-1] \Delta \tau)=-g_{0} \phi([n-1] \Delta \tau) \Delta \tau,
$$


whose discrete solution is

$$
\phi(n)=\left(1-g_{0} \Delta \tau\right)^{n} \phi(0) .
$$

Here $\phi(n)$ is the difference variable for a single individual chosen from the network at random and as $n \rightarrow \infty$ and $\Delta \tau \rightarrow 0$ such that clock time is $t=n \Delta \tau$ we have the apparently trivial result

$$
\phi(t)=e^{-g_{0} t} \phi(0) .
$$

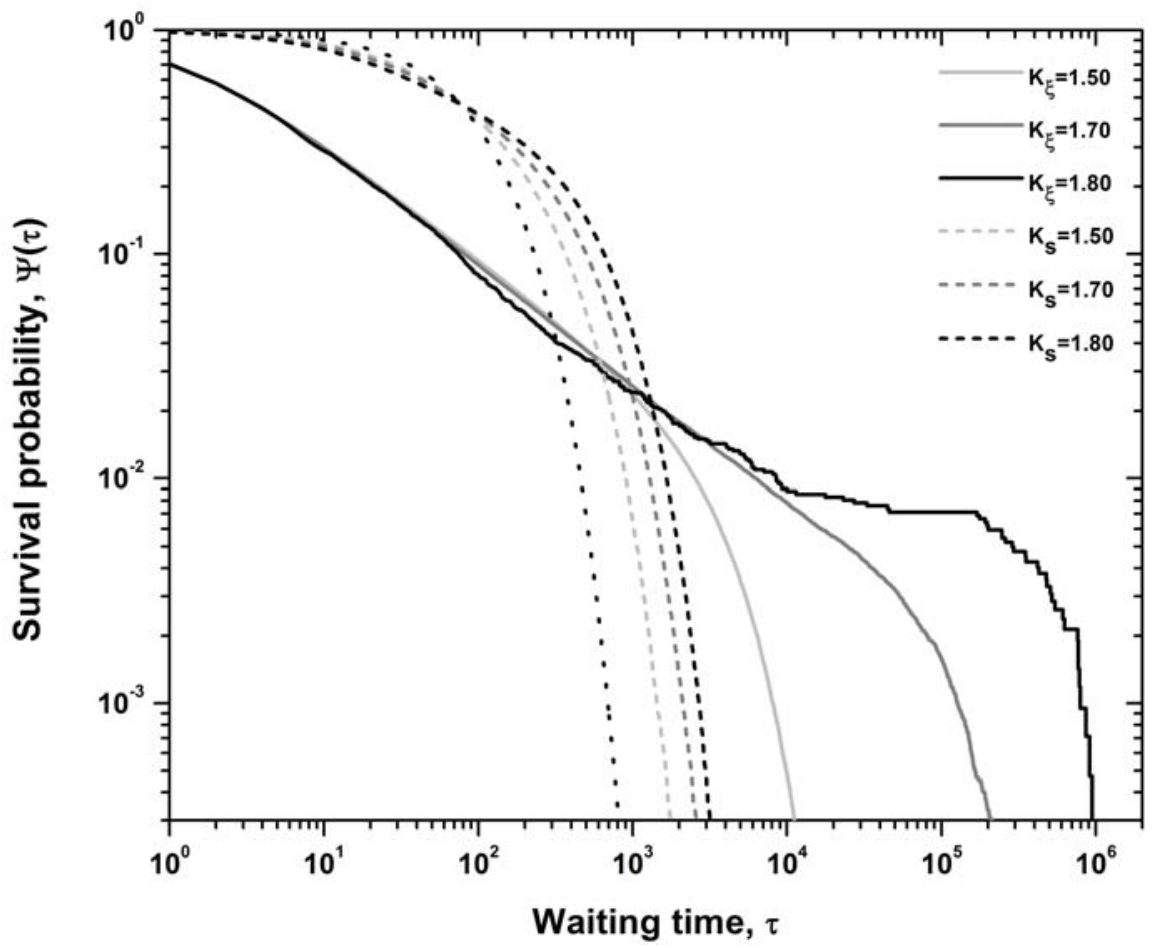

Figure 3: The survival probability $\Psi(t)$ of the global variable (solid lines) is compared with the transitions between two stable states for a single unit $s_{j}$ (dashed lines). Simulations were performed on a lattice of size $N=50 \times 50$ for $g_{0}=0.01$ and increasing values of the control parameter $\mathrm{K}$. The critical value of the control parameter is $K_{c}=1.72$. Dotted line corresponds to the exponential decay with rate $g_{0}$. The slope of the inverse power law region is $\mu-1=0.50$.

However when the individual is part of the network the exponential is not what is observed. The straightforward interpretation of the index $n$ given above is no longer correct. Instead there exists a stochastic connection between what is now the discrete operational time $n$ and the chronological or clock time $t$, 
one that is referred to as subordination in the mathematics literature [11]. We adopt the subordination interpretation here and assume that the time interval $t$ lies between the values $(n-1) \Delta \tau \leq t \leq n \Delta \tau$. Consequently the time $t$ is derived from a waiting-time $p d f$ given by that of the network as a whole:

$$
\psi(t)=\frac{(\mu-1) T^{\mu-1}}{(T+t)^{\mu}}
$$

where the survival probability as determined from Figure 3 to be $[1,2]$

$$
\Psi(t)=\int_{t}^{\infty} \psi\left(t^{\prime}\right) d t^{\prime}=\left(\frac{T}{T+t}\right)^{\mu-1} .
$$

An event occurring at time $n$ is an abrupt change from $\phi(n-1)$ to the state $\phi(n)$. At a generic time $t$ we can write the average probability difference as the generalized master equation (GME)

$$
\langle\phi(t)\rangle=\sum_{n=0}^{\infty} \int_{0}^{t} d t^{\prime} \psi_{n}\left(t^{\prime}\right) \Psi\left(t-t^{\prime}\right) \phi(n)
$$

where $\phi(n)$ is given by Eq.(15). Here we see that the GME replaces the twostate master equation of the DMM.

Note that $d t \psi_{n}(t)$ is the probability that $n$ events have occurred, the last one occurring in the time interval $(t, t+d t)$. The function $\Psi(t)$ denotes the probability that no event occurs up to time $t$ and is given by Eq.(18). The occurrence of an event corresponds to activating a decision with $\left(1-g_{0} \Delta \tau\right)$, so that activating $n$ such events transforms the initial condition $\phi(0)$ into the product $\left(1-g_{0} \Delta \tau\right)^{n} \phi(0)$. This form of the equation is kept from time $t^{\prime}$, at which time the last event occurs, up to time $t$, the time interval $t-t^{\prime}$ being characterized by no event occurring. Of course, the expression Eq.(19) takes into account that the number of possible events may range from the no-event case to that of infinitely many events. The conditions necessary for this result to occur are discussed by Svenkenson et al. [12].

It is useful to introduce Laplace variables in our discussion. The Laplace transform of a function $f(t)$ is denoted

$$
\widehat{f}(s) \equiv \int_{0}^{\infty} e^{-s t} f(t) d t
$$

Consequently using the convolution theorem Eq.(19) can be expressed in terms of Laplace transformed quantities

$$
\langle\widehat{\phi}(s)\rangle=\sum_{n=0}^{\infty} \widehat{\psi}_{n}(s) \widehat{\Psi}(s)\left(1-g_{0} \Delta \tau\right)^{n} \phi(0) \text {. }
$$


We assume the intervals between successive transitions are independent of one another so that the waiting time $p d f$ for $n$ transitions is the product of $n$ single transition $p d f$ 's:

$$
\widehat{\psi}_{n}(s)=[\widehat{\psi}(s)]^{n}
$$

and the Laplace transform of the survival probability is

$$
\widehat{\Psi}(s)=\frac{1}{s}[1-\widehat{\psi}(s)] .
$$

Inserting these last two expressions into Eq.(21) and evaluating the sum yields

$$
\langle\widehat{\phi}(s)\rangle=\frac{1}{s+g_{0} \Delta \tau \widehat{\Phi}(s)} \phi(0)
$$

whose inverse Laplace transform yields the GME:

$$
\frac{d\langle\phi(t)\rangle}{d t}=-g_{0} \Delta \tau \int \Phi\left(t-t^{\prime}\right)\left\langle\phi\left(t^{\prime}\right)\right\rangle d t^{\prime} .
$$

\subsection{Fractional Langevin Equation}

The function $\Phi(t)$ is a memory kernel containing the information on how the other elements in the network influence the dynamics of the individual element selected. The Laplace transform of the memory kernel is

$$
\widehat{\Phi}(s)=\frac{s \widehat{\psi}(s)}{1-\widehat{\psi}(s)}
$$

and a complete discussion of its properties is now given in textbooks [3].

Previous analysis has shown that the waiting-time $p d f$ is an inverse powerlaw distribution. The asymptotic behavior of the GME is determined by considering the waiting-time $p d f$ given by Eq.(17) as $s \rightarrow 0$ :

$$
\widehat{\psi}(s) \approx 1-\Gamma(2-\mu)(s T)^{\mu-1}
$$

so that Eq.(24) reduces to

$$
\langle\widehat{\phi}(s)\rangle=\frac{1}{s+\lambda^{\mu-1} s^{2-\mu}} \phi(0)
$$

and the rate parameter has the value

$$
\lambda^{\mu-1}=\frac{g_{0} \Delta \tau}{\Gamma(2-\mu) T^{\mu-1}} ; 1<\lambda<2 .
$$

We now assume that the exact equation for the single person dynamics has both an average and a fluctuating part just as we found in the mean field treatment of 
the double well potential $[1,8]$. Consequently in terms of the Laplace variables we have the stochastic equation

$$
\widehat{\phi}(s)=\frac{1}{s+\lambda^{\mu-1} s^{2-\mu}} \phi(0)+\frac{1}{s+\lambda^{\mu-1} s^{2-\mu}} \widehat{\varepsilon}(s),
$$

which has the inverse Laplace transform

$$
D_{t}^{\mu-1}[\phi(t)]-\frac{t^{1-\mu}}{\Gamma(2-\mu)} \phi(0)=-\lambda^{\mu-1} \phi(t)+\varepsilon(t) .
$$

This is a stochastic fractional master equation or fractional Langevin equation (FLE) in which the stochastic properties of $\varepsilon(t)$ are determined by the fluctuations of the complex social network. The fractional derivative in this equation is of the Riemann-Liouville form [?]

$$
D_{t}^{q}[f(t)] \equiv \frac{1}{\Gamma(m-q)} \frac{d^{m}}{d t^{m}} \int_{0}^{t} \frac{f\left(t^{\prime}\right) d t^{\prime}}{\left(t-t^{\prime}\right)^{q-m+1}}
$$

due to the global dynamics of the network $q=\mu-1>0$, with the requirement that $m$ is the smallest integer such that $m-1<q<m$ so that $m=1$ in Eq.(32).

The solution to the FLE is given by

$$
\begin{aligned}
\phi(t)= & \phi(0) E_{\mu-1}\left(-(\lambda t)^{\mu-1}\right) \\
& +\int_{0}^{t}\left(t-t^{\prime}\right)^{\mu-2} E_{\mu-1, \mu-1}\left(-\left(\lambda\left[t-t^{\prime}\right]\right)^{\mu-1}\right) \varepsilon\left(t^{\prime}\right) d t^{\prime} .
\end{aligned}
$$

where the homogeneous solution to the fractional equation is the Mittag-Leffler function (MLF)

$$
E_{\theta}\left(-z^{\theta}\right)=\sum_{k=0}^{\infty} \frac{(-1)^{k} z^{k \theta}}{\Gamma(1+k \theta)} ; \quad \theta>0
$$

and the kernel of the integral is in terms of the second order MLF taken up in the next section.

It is useful to replace Eq.(30) with the Laplace transform of the singleelement autocorrelation function

$$
\widehat{C}(s) \equiv \frac{E[\langle\widehat{\phi}(s)\rangle \phi(0)]}{E\left[\phi(0)^{2}\right]}=\frac{1}{s+\lambda^{\mu-1} s^{2-\mu}}
$$

where $E[\cdot]$ is an average over an ensemble of initial states. Again assuming the existence of fluctuations and rearranging the terms in Eq.(35), the inverse 
Laplace transform [?] of the resulting equation yields the fractional differential equation for the autocorrelation function

$$
D_{t}^{\mu-1}[C(t)]-\frac{t^{1-\mu}}{\Gamma(2-\mu)}=-\lambda^{\mu-1} C(t)+\frac{E[\varepsilon(t) \phi(0)]}{E\left[\phi(0)^{2}\right]} .
$$

It is clear that the solution to the homogeneous fractional equation is again the MLF, but the behavior of the individual autocorrelation function also depends on the the properties of the random fluctuations as we show in the next section.

\section{Fractional Dynamics and Complex Networks}

The solution to the GME is obtained from the exact Laplace transform equation Eq.(24). However it is notoriously difficult to obtain analytic expressions by direct inversion of the resulting equations due to the complexity of the exact form of the Laplace transform memory kernel. Consequently, the strategy is to consider the asymptotic forms of the solution, which we do by examining the solutons to the FLE. We find that the properties of the fluctuations change as the control parameter is varied from the subcritical, critical and supercritical regions.

\subsection{Subcritical Solution}

The analytic solution to Eq.(31) with the noise set to zero is obtained elsewhere [?] by inverse Laplace transforming Eq.(28). The solution is the MLF, as we said,

$$
\phi(t)=\phi(0) E_{\mu-1}\left(-[\lambda t]^{\mu-1}\right)
$$

The autocorrelation function for the single individual, the solution to the fractional differential equation Eq.(36), is therefore also given by the MLF

$$
C(t)=E_{\mu-1}\left(-[\lambda t]^{\mu-1}\right) .
$$

From the series form of the MLF it is evident that for $\mu=2$ the autocorrelation function would be an exponential. Consequently the influence of the network on the behavior of the individual in this case would be essentially that of uncorrelated random noise and therefore would not qualitatively change from the Poisson nature of an isolated individual. However this is not the case for other values of the inverse power-law index. The dynamic behavior of the network results in a stretched exponential autocorrelation for the dynamics of the individual

$$
\lim _{t \rightarrow 0} C(t)=1-\frac{(\lambda t)^{\mu-1}}{\Gamma(\mu)} \approx \exp \left(-\frac{[\lambda t]^{\mu-1}}{\Gamma(\mu)}\right)
$$


Note that the early time behavior of the MLF is the indicated stretched exponential.

The ten individual elements used in the evaluation of the single autocorrelation functions were selected at random from a DMM network calculation on a two-dimensional lattice of size $100 \times 100$ with nearest neighbor interactions. It is clear from the autocorrelation functions depicted in Figure 4 that in the subcritical regime the data are not indicative of exponential decay. For the subcritical case of $K=1.50$ the stretched exponential function in Eq.(39) gives a remarkably good fit to the autocorrelation of the time series data. The MLF index is seen to fall in the interval $1.43 \leq \mu \leq 1.55$, the rate of decay of the stretched exponential is in the range $3.4 \times 10^{-3} \leq \lambda \leq 4.8 \times 10^{-3}$, and the quality of fits all lie in the interval $0.97 \leq r^{2} \leq 0.99$. The fitting of the analytic autocorrelation function at early time to the numerically generates curves using the DMM is certainly very good in the subcritical domain.

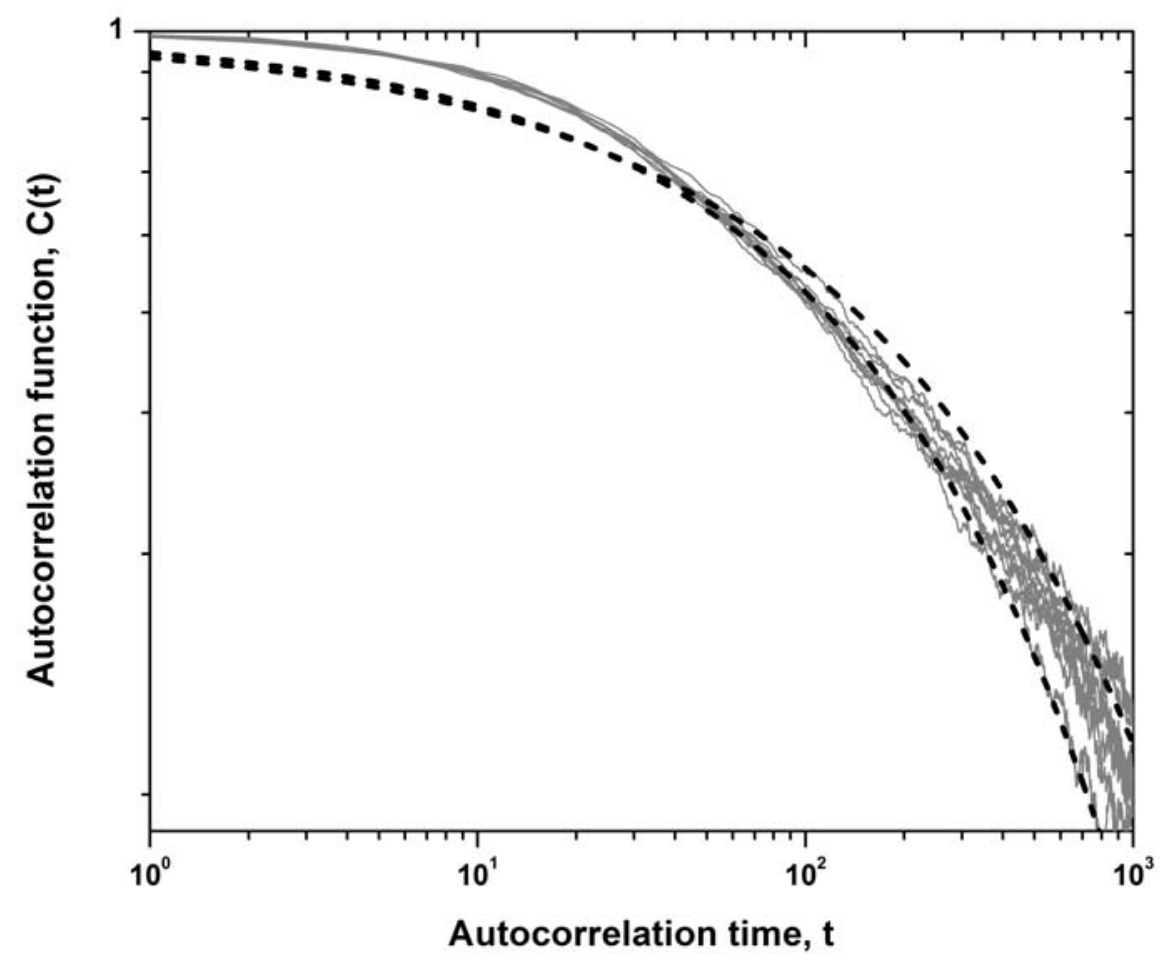

Figure 4: Ten realizations of the single element autocorrelation function, each selected randomly from the elements on a two-dimensional lattice, under DMM dynamics with $N=100 \times 100, g_{0}=0.01$ and $K=1.50$ are plotted with thin gray lines. Thick dashed lines denote the fit with a stretched exponential function. The fits to the most upper and lower single element autocorrelation functions are shown. 


\subsection{Supercritical Solutions}

In the supercritical region of the control parameter the single elements used in the evaluation of the autocorrelation function were selected at random from the same DMM network calculations as those above. The calculations were done on a $100 \times 100$ two-dimensional lattice with nearest neighbor interactions. It is evident from the autocorrelation functions depicted in Figure 5 that there exists a wide spread in either the kind of distributions that characterize the network dynamics in this region or there is an equally wide spread in the parameter values required to fit the data. Let us consider the last alternative first.

We temporarily assume that the network dynamics in the supercritical region is similar to that in the subcritical region. We mean by this that the stretched exponential can be used to describe the autocorrelation function in both domains and therefore each of the data curves in Figure 5 can be fit using Eq.(39) albeit with a wide distribution of rates and exponents. The quality of the fit is $r^{2} \geq$ 0.84 for each of the curves in Figure 5, which is remarkably good given the vertical spread in the distal values of the autocorrelation function curves. In many fits to social data the $r^{2}$ would be sufficient to draw a positive conclusion. However here we investigate the influence of the noise produced by the network dynamics.

We assume that the random fluctuations $\varepsilon(t)$ in the FLE have Gaussian statistics and are exponentially correlated with the initial condition such that

$$
E[\phi(0) \varepsilon(t)]=2 E\left[\phi(0)^{2}\right] D e^{-\gamma t} .
$$

In addition we insert the identity

$$
\frac{d E_{\mu-1}\left(-(\lambda t)^{\mu-1}\right)}{d t}=-\lambda^{\mu-1} t^{\mu-2} E_{\mu-1, \mu-1}\left(-(\lambda t)^{\mu-1}\right)
$$

into Eq.(33) to obtain for the autocorrelation function

$$
C(t)=E_{\mu-1}\left(-(\lambda t)^{\mu-1}\right)-\frac{D}{\lambda^{\mu-1}} \int_{0}^{t} \frac{d E_{\mu-1}\left(-\left[\lambda\left(t-t^{\prime}\right)\right]^{\mu-1}\right)}{d t^{\prime}} e^{-\gamma t^{\prime}} d t^{\prime}
$$

Integrating Eq.(41) by parts and using the method of steepest decent on the remaining integral yields for the early time solution

$$
\begin{aligned}
\lim _{t \rightarrow 0} C(t)= & \frac{D_{j}}{\lambda^{\mu-1}}+\left(1-\frac{D}{\lambda^{\mu-1}} e^{-\gamma t}\right) \exp \left[-\frac{(\lambda t)^{\mu-1}}{\Gamma(\mu)}\right] \\
& -\frac{\gamma D}{\lambda^{\mu-1}} e^{F\left(t^{*}\right)} e^{-\gamma t} \operatorname{erf}\left[\sqrt{2\left|F^{\prime \prime}\left(t^{*}\right)\right| t}\right]
\end{aligned}
$$




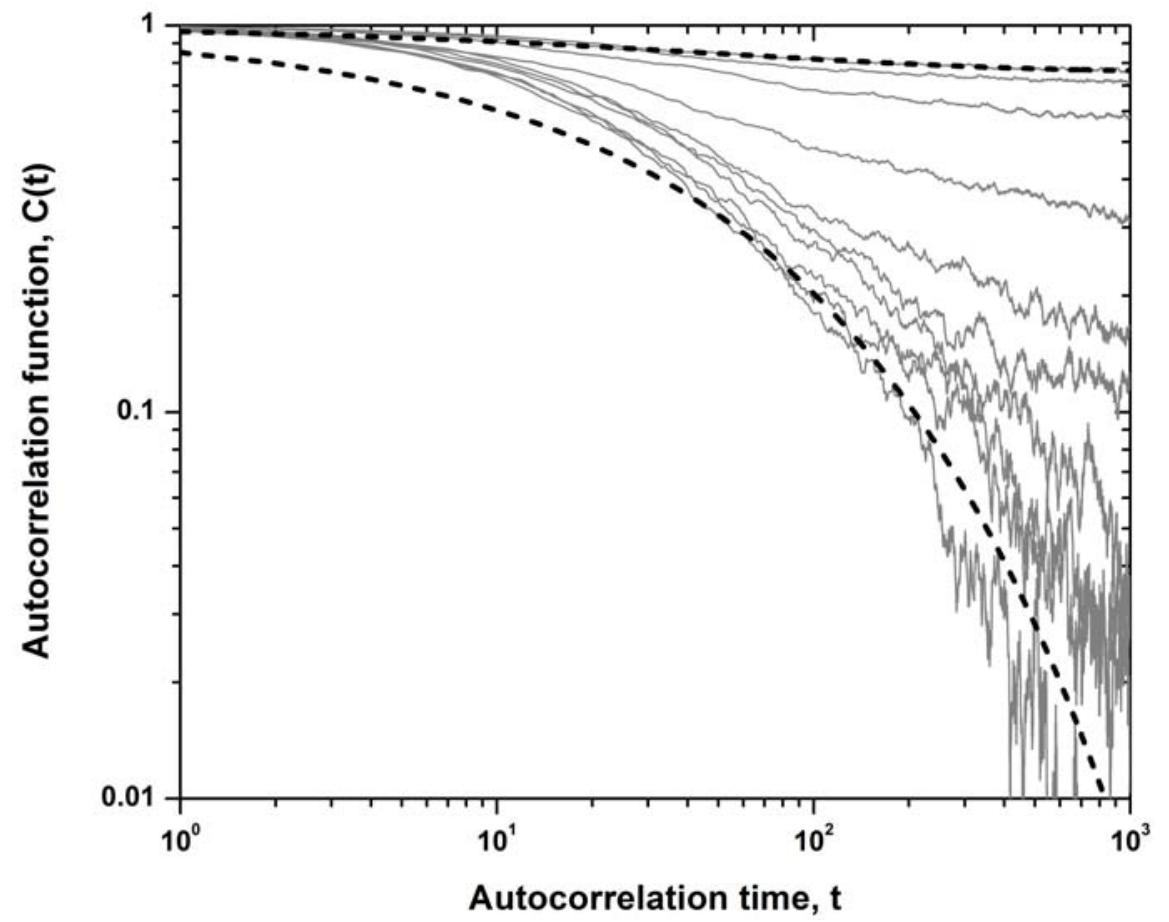

Figure 5: The autocorrelation functions (thin gray lines) are calculated for each of 100 time series obtained from a DMM calculation on a two-dimension lattice for a control parameter greater than the critical value $K=1.9>K_{c}$. There are ten time series for each of ten individuals chosen at random from the network. Thick dashed lines denote the fit with Eq.(44). The most upper and lower single node autocorrelation function has been fitted.

and the new functions are

$$
F\left(t^{*}\right)=\left[\left(\frac{\gamma}{\lambda(\mu-1)}\right)^{\mu-1} \Gamma(\mu)\right]^{\frac{1}{\mu-2}} ; \quad F^{\prime \prime}\left(t^{*}\right)=(2-\mu)\left[\frac{\gamma \Gamma(\mu)}{\lambda^{\mu-1}(\mu-1)}\right]^{\frac{1}{2-\mu}} .
$$

We approximate the early time solution to be

$$
\lim _{t \rightarrow 0} C(t) \approx \frac{D}{\lambda^{\mu-1}}+\left(1-\frac{D}{\lambda^{\mu-1}} e^{-\gamma t}\right) \exp \left[-\frac{(\lambda t)^{\mu-1}}{\Gamma(\mu)}\right]
$$

where the last term in Eq.(42) has been dropped. We subsequently use the fitted parameter values to calculate the size of the neglected term, which is determined to be orders of magnitude smaller than the terms retained and therefore its neglect is justified. 
Equation (44) is used to fit all ten curves in Figure 5 using the exponent $\mu=1.5$ and dissipation rate $\lambda=0.02$. The quality of the fit to all the curves on the graph for the empirical strength of the fluctuations $D$ and the decay rate of the fluctuations $\gamma$ for the individuals depicted in Figure ?? fall in the range $0.96 \leq r^{2} \leq 0.99$. For weakly decaying fluctuations the individual autocorrelation is high as is the strength of the fluctuations; this is the region of exponentially truncated stretched exponential autocorrelation. For rapidly decaying fluctuations the individual autocorrelation decays as a stretched exponential. The fitting of the approximate analytic solution to the autocorrelation function to the numerically generated curves using the DMM is certainly very good in the supercritical domain.

\subsection{Critical Solutions}

As the critical point is approached from the subcritical region the autocorrelation function changes as would be expected due to the formation of clusters as the network undergoes a phase transition. The plunging stretched exponential that was observed in the subcritical region as seen in Figure 4 is replaced with a more gently decreasing correlation. Figure 6 depicts another ten randomly selected single element trajectories from DMM calculations on the twodimensional lattice. In this case the control parameter has the critical value 1.70 and the autocorrelation function is calculated from the single particle trajectories. It is evident by comparing these data with the data curves in Figure 4 that the autocorrelation does not decrease as quickly in time and there is more variability among the ten slopes at late time.

The question is whether the MLF solution to the FLE can be used to fit these data as well as it did the subcritical data or whether we require an additional mechanism such as the fluctuations in the supercritical region. We find that at early times the stretched exponential approximation to the MLF fits the data very well as shown in Figure 6. At late times correlated fluctuations generated by the remainder of the network must be included.

The data curves in Figure 6 are extremely well fit using the stretched exponential function at early times, however, the inverse power law at late times using the same MLF is not a good fit. The fits by separate asymptotic solutions to the data in the critical region are excellent each with $r^{2}>0.99$. However there is a problem and that is the low value of the inverse power-law index, it is 1.22 rather than the 1.5 found in the numerical calculation of the survival probability for the network and used in the subordination process. This change of inverse power-law index suggests that the dynamic equation for the autocorrelation function is given by Eq.(30) but the noise is no longer given by exponentially decaying fluctuations. The strength of the fluctuations at the critical points are assumed to decay as an inverse power law:

$$
E[\phi(0) \varepsilon(t)]=E\left[\phi(0)^{2}\right] \bar{D}\left(\frac{T}{T+t}\right)^{\nu}
$$




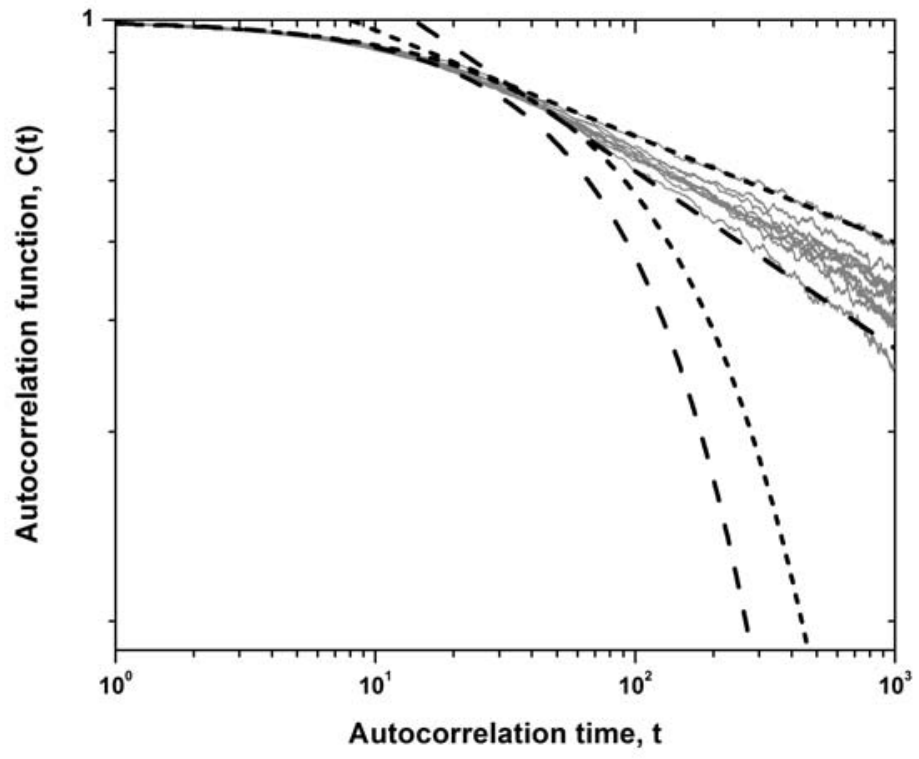

Figure 6: Ten realizations of the single element autocorrelation function selected randomly from the two-dimensional lattice with $N=100 \times 100, g_{0}=0.01$ and $K=1.70 \approx K_{c}$. Thick dashed lines denote the fit with an inverse power law function. The most upper and lower single node autocorrelation function has been fitted.. The data are fit to a stretched exponential at early time (dashed light gray line) with $\lambda_{0} \approx 0.01$ and to an IPL at late times (dashed dark gray line) with index $\mu-1 \approx 0.22$. The quality of fit $r^{2} \geq 0.99$ for all ten curves in both regions.

so that the Laplace transform of the autocorrelation function is

$$
\widehat{C}(s)=\frac{1}{s+\lambda^{\mu-1} s^{2-\mu}}+\frac{\bar{D}}{s+\lambda^{\mu-1} s^{2-\mu}} \frac{\Gamma(1-\nu) T}{(s T)}\left[e^{s T}-E_{1-\nu}^{s T}\right] .
$$

The time asymptotic autocorrelation function is obtained by considering the $s \rightarrow 0$ limit and noting $1>2-\mu$ as well as $\nu-1<0$,

$$
\begin{aligned}
\lim _{s \rightarrow 0} \widehat{C}(s) & =\frac{1}{s+\lambda^{\mu-1} s^{2-\mu}}\left[1+\Gamma(1-\nu) \bar{D}(s T)^{\nu-1}\right] \\
& \approx \frac{\Gamma(1-\nu) \bar{D} T^{\nu-1}}{\lambda^{\mu-1}} s^{\mu+\nu-3}
\end{aligned}
$$


and consequently yields the inverse power law with a modified index

$$
\lim _{t \rightarrow \infty} C(t)=\frac{\Gamma(1-\nu) \bar{D}}{\Gamma(4-\mu-\nu)(\lambda T)^{\mu-1}}\left(\frac{T_{j}}{t}\right)^{\mu+\nu-2} .
$$

From the fits to the curves in Figure 6 we determine that the average fluctuation relaxation rate to be $\nu \approx 0.72$ with a quality of fit $r^{2} \geq 0.99$ for each of the ten curves considered.The relaxation properties of the fluctuations are therefore seen to dominate the autocorrelation of the individual elements of the network at late times and the analytic fit in the two time regimes are uniformly excellent.

\section{Conclusion}

The numerical solution of the DMM on a $100 \times 100$ lattice with elements at each of the nodes and with nearest neighbor interactions gives rise to an inverse power-law survival probability for the global variable introduced in Section 2. Using the notion of a subordination time, that being the time experienced by an individual, with the influence of the network entering into the individual's dynamics through the distribution of critical events, the dynamics of an individual is determined by a fractional master equation. The explicit form of the FLE depends on whether the network dynamics is in the subcritical, critical or supercritical domains. Only in the subcritical domain is the solution to the FLE, that being the MLF, sufficient to describe the dynamic response of an individual to the other 9,999 dynamic elements of the network. In the supercritical and critical domains the FLE is modified to include a correlated stochastic driver. In the supercritical domain the relaxation of the fluctuations is exponential and solution to the stochastic FLE fits the individual autocorrelation calculated data remarkably well. In the critical domain the relaxation of the fluctuations is modeled by an inverse power law and the solution to the stochastic FLE again fits the individual autocorrelation data surprisingly well.

The lesson to be learned from this combination of computation and analysis presented herein is that complex networks of finite size whose dynamics are members of the Ising universality class described by the DMM have an analytic not just a numerical description. Instead of confining the dynamic description to that of the macroscopic variable, that being the global or average state of the network, one can also investigate how individual members of the network respond to the influence of the network as a whole. If we consider the fluctuations in the global dynamics to be microscopic, and the potential of the global variable to be macroscopic, then the real time dynamic description of the individuals is mesoscopic. In general the mesoscopic dynamics have a fractional stochastic differential representation. 


\section{Acknowledgement}

The authors warmly thank the Army Research Office for financial support of this research.

\section{References}

[1] S, Bianco, E. Geneston, P. Grigolini and M. Ignaccolo, "Renewal aging as emerging property of phase synchronization", Physica A 387, 1387 (2008).

[2] M. Turalska, M. Lukovic, B.J. West, and P. Grigolini, "Complexity and synchronization", Phys. Rev. E 80, 021110(2009)

[3] B.J. West and P. Grigolini, Complex Webs, Cambridge University Press, UK (2011).

[4] B.J. West and M. Turalska, "Network of Echoes", to appear in Chaos, Solitons 83 Fractals (2013).

[5] C.A. Ellwood, "The Theory of Imitation in Social Psychology", Am. J. Sociol. 6, 721-741 (1901).

[6] J.M. Baldwin, Mental Development in the Child and the Race (1895); Social and Ethical Interpretations in Mental Development (1897).

[7] M.G. Tarde, Les Lois de l'Imitation (1890); La Logique sociale (1895); Les Lois socials (1898).

[8] M. Turalska, B.J. West, P. Grigolini, "Temporal complexity of the order paramerer at the phase transition", Phys. Rev. E 83, 061142 (2011).

[9] R. Zwanzig, Nonequilibrium Statistical Mechanics, Oxford University Press, NY (2001).

[10] P. Grigolini, A. Rocco and B.J. West, "Fractional calculus as a macroscopic manifestation of randomness", Phys. Rev. E 59, 2603 (1999).

[11] W. Feller, An Introduction to Probability Theory and Its Applications, John Wiley, NY (1966).

[12] A. Svenkeson, M.T. Beig, M. Turalska, B.J. West and P. Grigolini, "Fractional Ecology: Decorrelation versus Friction", under review 